\title{
GASTROINTESTINAL MUCORMYCOSIS OF NEONATE MASQUERADING AS NECROTISING ENTEROCOLITIS
}

\author{
Shenbagam Jeevakarunyam Muthukanagarajan1, Indumathi Karnan², Pappathi Sadagopan³, Saraswathi Manickam
}

1 Post Graduate, Department of Pathology, Institute of Pathology, Madras Medical College, Chennai.

${ }^{2}$ Assistant Professor, Department of Pathology, Institute of Child Health, Madras Medical College, Chennai.

3Professor, Department of Pathology, Institute of Child Health, Madras Medical College, Chennai.

${ }^{4}$ Director, Department of Pathology, Institute of Pathology, Madras Medical College, Chennai.

\begin{abstract}
Mucormycosis is a rare opportunistic invasive fungal infection that seldom infects healthy persons. Mucormycosis can affect any organ in the body among which gastrointestinal mucormycosis is very rare. Antemortem or preoperative diagnosis of gastrointestinal mucormycosis is not possible until histopathological demonstration of the fungal elements is done. Treatment consists of surgical debridement with systemic antifungal therapy with amphotericin B. The high mortality rate associated with GIMN (Gastrointestinal Mucormycosis of Neonate) is not only due to delay in diagnosis, but also because of inadequate and inappropriate treatment. All the infants with bowel surgery done for perforating or inflammatory lesions should be subjected to careful histopathological examination, else atypical causes for bowel perforations like GIM might be missed. Herewith presenting a rare case of gastrointestinal mucormycosis that occurred in a full-term, 5-day-old immunocompetent neonate.
\end{abstract}

\section{KEYWORDS}

GIMN, Gastrointestinal Mucormycosis, Necrotizing Enterocolitis.

HOW TO CITE THIS ARTICLE: Muthukanagarajan SJ, Karnan I, Sadagopan P, et al. Gastrointestinal mucormycosis of neonate masquerading as necrotising enterocolitis. J. Evolution Med. Dent. Sci. 016;5(46):2941-2943, DOI: 10.14260/jemds/2016/684

\section{INTRODUCTION}

Mucormycosis is a rare opportunistic invasive fungal infection affecting immunocompromised patients.(1) Mucormycosis can affect any organ in the body, among which gastrointestinal mucormycosis is very rare. There has been an increase in number of these cases over the past decade.(2) The antemortem or preoperative diagnosis of Gastrointestinal Mucormycosis of Neonate (GIMN) is an exception, as in most cases the diagnosis is retrospective made after death or discharge of the neonate.(3) Herewith presenting a rare case of gastrointestinal mucormycosis that occurred in a full-term 5-day-old immunocompetent neonate.

\section{CASE REPORT}

A full-term female neonate was delivered by a primi gravida by emergency caesarean section due to history of prolonged labour. There was no history of any maternal risk factors. The baby weighed $2180 \mathrm{~g}$. The infant did not cry after birth and did not move any of her limbs. Apgar score at 1 and 5 minutes were $3 / 10$ and $4 / 10$, respectively. The infant was shifted to intensive care unit and was kept under incubator. CT brain showed hypoxic-ischaemic encephalopathy changes. On day 5 of life, the infant developed abdominal distension and bowel sounds were sluggish. Nasogastric intubation revealed bilious aspirate. X-ray abdomen showed distended bowel loops without any evidence of pneumatosis intestinalis or pneumoperitoneum. All the other investigations done ruled out presence of congenital anomalies or obstructive causes of abdominal distension. Serum CRP levels were elevated.

Financial or Other, Competing Interest: None.

Submission 22-04-2016, Peer Review 19-05-2016,

Acceptance 24-05-2016, Published 09-06-2016.

Corresponding Author:

Shenbagam Jeevakarunyam Muthukanagarajan,

Plot Number-117, 118,

P.U. Chinnapa Nagar,

Pudukottai-622001,

Tamilnadu.

E-mail: shenbajm9@gmail.com

DOI: $10.14260 /$ jemds/2016/684
Necrotising enterocolitis was suspected and the infant was put on intravenous antibiotics such as ceftriaxone, amikacin and metronidazole. General condition of the infant showed no improvement and the antibiotic was changed to piperacillin-tazobactam with meropenem. On day 9 of life, the infant developed rectal bleeding. Since there was clinical deterioration of the neonate and there was no response to antibiotics, surgical exploration was planned. Per-operatively, the bowel was gangrenous, bile-stained and colonic and ileal perforations were made out. Affected segments of colon and ileum were resected and ileostomy was performed.

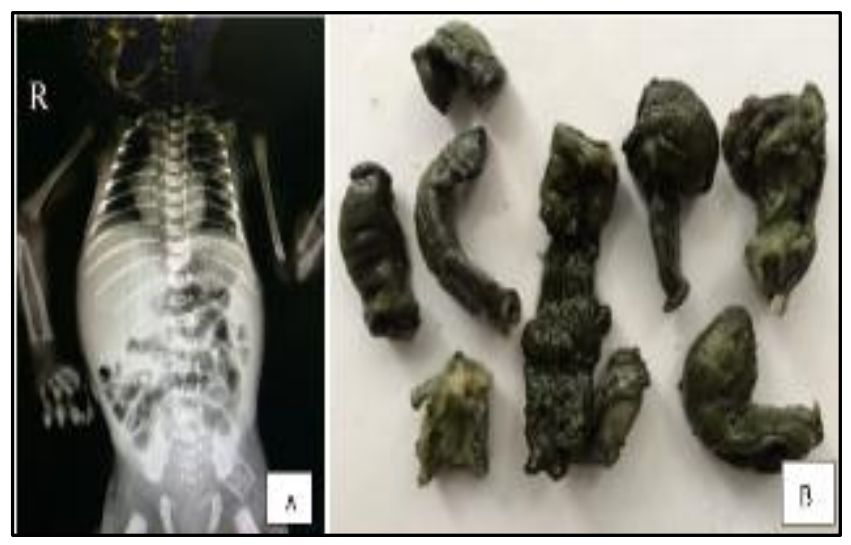

Fig. 1: A. X-ray Abdomen showing Distended Bowel Loops Without Evidence of Pneumatosis Intestinalis \&

Pneumoperitoneum, B. Resected Bowel Fragments showing Bile Stained and Gangrenous Colonic and Ileal Segments

Microscopic examination of the bowel segments showed only few viable foci of small intestinal and colonic mucosa with predominant areas showing transmural necrosis of intestinal wall. Seen admixed are numerous broad aseptate fungal hyphae exhibiting right angled branching. There was intense neutrophilic infiltration and focal areas of multinucleated giant cell reaction. Diagnosed as Gastrointestinal Mucormycosis of Neonate (GIMN) 


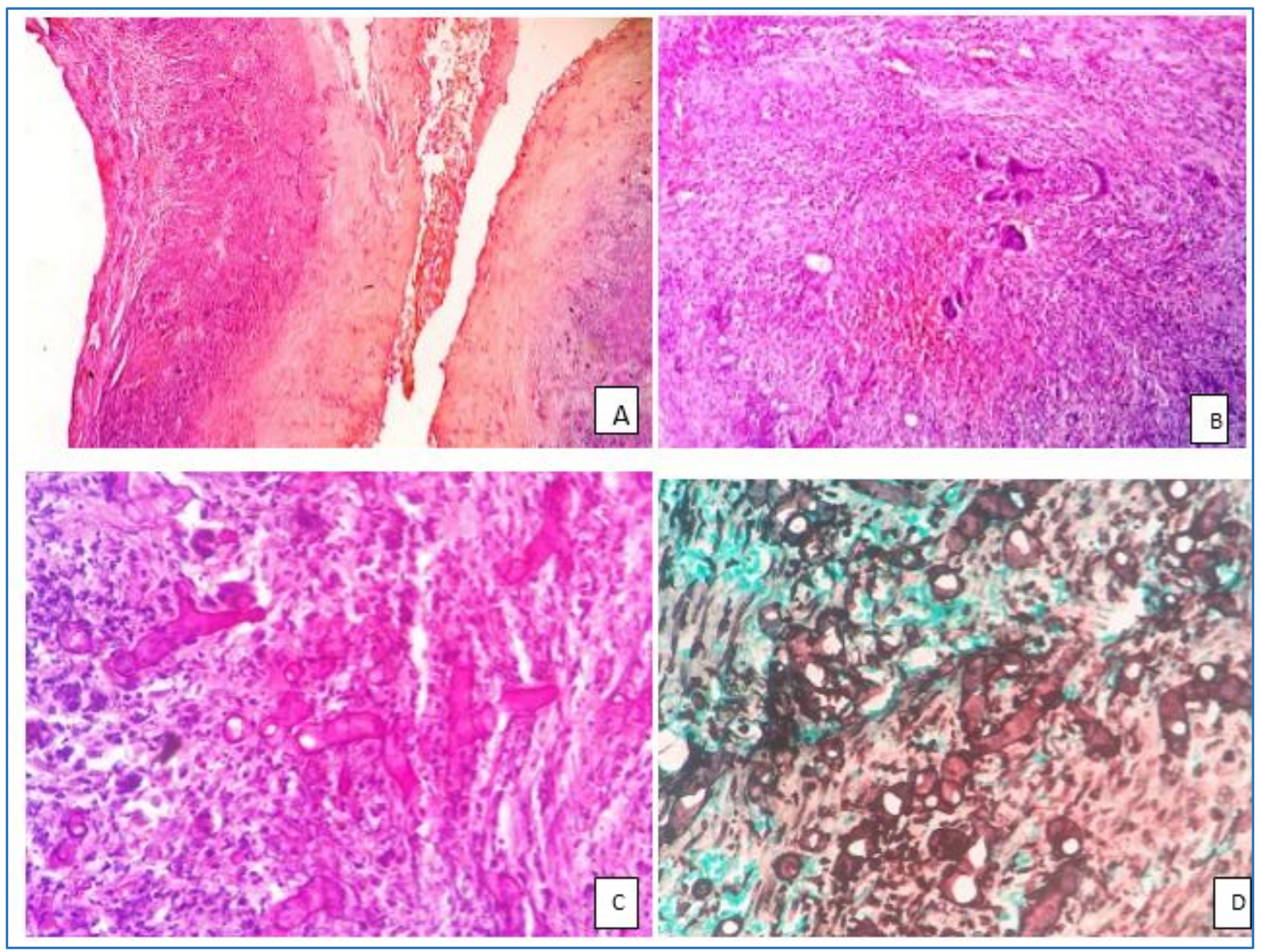

Fig. 2: A. Sections from Colonic Segment showing Transmural Necrosis of Bowel Wall (H\&E, 40X), B. Focal Areas showing Multinucleated Giant Cell Reaction (H\&E, 100X), C. Broad Aseptate Fungal Hyphae Exhibiting Right Angled Branching with Surrounding Areas showing Intense Neutrophilic Infiltration and Necrosis (H\&E, 400X), D. Gomori Methenamine Silver Stain Highlighting the Fungal Hyphae (GMS, 400X)

\section{DISCUSSION}

Mucormycosis is a saprophytic and ubiquitous fungi (4) that belongs to class zygomycetes.(5) It most commonly involves nasal and paranasal sinuses, brain and orbits. Gastrointestinal mucormycosis is very rare. Its incidence is underestimated since all those affected infants whether died or survived might have not had detailed histopathological examination.(4) and those infants suspected to have NEC but died in spite of appropriate therapy were labelled as refractory or fulminant NEC.(3) without any histopathological evaluation of these cases. All these cases might account to the pool of underestimated GIMN (Gastrointestinal Mucormycosis of Neonate) cases. (3)

GIMN is a rarest presentation of mucormycosis accounting for $7 \%$ of cases.(1) Infection is acquired by inoculation, inhalation or ingestion of fungal spores and the route of entry of the fungal spores determines the site of infection.(4) The most commonly involved sites includes stomach, colon and ileum with neonates showing predominant involvement of colon.(5) The affected neonate presents with abdominal distension, nausea and vomiting. Haematochezia and fever may also occur.(2) Many predisposing factors are held responsible for the occurrence of GIMN. Those involved in this particular case includes nasogastric intubation, intravenous broad-spectrum antibiotics, neonate treated in intensive care units and systemic hypoxia. The other predisposing factors are preterm baby, steroid therapy, malnutrition, immunosuppression, low birth weight, immature immune system of the neonate with fragile skin barrier, orogastric intubation. $(1,5,6,7,8)$

Members of mucoraceae show affinity for arterial invasion leading to thrombosis and ischaemic infarction of the tissue supplied by the artery. In gastrointestinal mucormycosis, this leads to gangrene of the involved bowel segment mimicking NEC (Necrotising Enterocolitis).(3) which is considered as the closest differential diagnosis of GIMN.(9) Absence of response to antibiotic therapy, absence of pneumatosis intestinalis, presence of fungal hyphae and thrombosis of small vessels favours gastrointestinal mucormycosis.(1) Necrotising enterocolitis occurs due to reperfusion injury to the bowel as a result of which the affected bowel segments will be moist, congested and will undergo putrefaction. On the contrary gastrointestinal mucormycosis occurs due to vascular thrombosis of the affected mesenteric artery leading to dry, avascular bowel and it will undergo infarction.

The affected bowel adheres to the adjacent healthy bowel segment to imbibe oxygen. Therefore, cases of GIMN presents 
with an early palpable abdominal mass and abdominal distension rather than bowel perforation, rectal bleeding or pneumoperitoneum. But there were reports of few cases of GIMN presented with rectal bleeding, pneumoperitoneum and pneumatosis intestinalis. This lead to identification of 2 forms of GIMN - Primary and Secondary. Primary GIMN caused by a direct fungal colonisation of bowel by mucormycosis. This form of GIMN is very rare. In secondary GIMN, fungal invasion occurs following a predisposing NEC of the bowel. The devitalised tissue of NEC provides a fertile medium for the growth of the fungus. Hence, the cases of secondary GIMN presents with mixed clinical features of both GIMN and NEC.(3)

It is necessary to differentiate GIMN from simple fungal contamination.

This can be done by demonstrating fungal hyphae in viable tissue with aid of special stains like Gomori Methenamine Silver (GMS) and Periodic Acid Schiff (PAS).(3) Fungal cultures are positive in only $33 \%$ of cases and are time consuming. Hence, most authors suggests histopathological study rather than mycological cultures for diagnosing gastrointestinal mucormycosis.(3) Angio-invasion and thrombosis of vessels by mucormycosis hinders the drug delivery to the affected tissue.(10) Hence, surgical debridement of affected tissue together with adequate antifungal treatment with amphotericin $\mathrm{B}$ forms the mainstay of treatment.(3) It has been shown that the fungicidal activity of amphotericin B has been enhanced by rifampicin.(11) The mortality rate in GIMN is more than $75 \%$.(3) This high mortality rate is not only due to delay in diagnosis, but also because of inadequate and inappropriate treatment.(12) From the index case diagnosed in 1994, till date 30 cases of GIMN were reported, of which 22 cases were from India. Of all the diagnosed cases, only 14 survived.(1)

Timely intervention with parenteral amphotericin B in surgically treated cases suspected to have necrotising enterocolitis might reduce the hidden mortality due to fungal sepsis and all infants with bowel surgery done for perforating or inflammatory lesions should be subjected to careful histopathological examination, else atypical causes for bowel perforations like GIM might be missed.(4)

\section{CONCLUSION}

One of the most important factors contributing to the high mortality associated with gastrointestinal mucormycosis is lack of clinical suspicion. Therefore, the clinicians and pathologists should have a strong suspicion of gastrointestinal mucormycosis in those neonates clinically suspected as necrotising enterocolitis, but showing no response to antibiotics. Earlier the diagnosis, better the survival rate.

\section{REFERENCES}

1. Pragati Aditya Sathe, Ratnaprabha Kundlikrao Ghodke, Bhuvaneshwari Mahendra Kandalkar. A survivor of neonatal intestinal mucormycosis. Journal of Clinical and Diagnostic Research 2015;9(8):ED24-5.

2. Brad Spellberg. Gastrointestinal mucormycosis: an evolving disease. Gastroenterology \& Hepatology 2012;8(2):140-2.

3. Raveenthiran V. Gastrointestinal mucormycosis mimicking necrotizing enterocolitis of newborn. Journal of Neonatal Surgery 2013;2(4):41.

4. Siu KL, Lee WH. A rare cause of intestinal perforation in an extreme low birth weight infant $\mathrm{F}$ gastrointestinal mucormycosis: a case report. Journal of Perinatology 2004;24:319-21.

5. Recep Tekin, Ozben Yalcın, Sirin Menekse Yılmaz, et al. Gastrointestinal mucormycosis causing an acute intestinal obstruction in neonate patient. Journal of Microbiology and Infectious diseases 2011;1(1):35-7.

6. Patra S, Vij M, Chirla DK, et al. Unsuspected invasive neonatal gastrointestinal mucormycosis: aclinicopathological study of six cases from a tertiary care hospital. J Indian Assoc Paediatr Surg 2012;17(4):153-6.

7. Jain D, Kohli K. Neonatal gastrointestinal mucormycosis clinically mimicking necrotizing enterocolitis. Eur J Paediatr Surg 2009;19(6):405-7.

8. Sarin YK. Intestinal mucormycosis in a neonate: a case report and review. J Indian Assoc Paediatr Surg 2010;15(3):98-100.

9. Dhingra KK, Mandal S, Khurana N. Unsuspected intestinal mucormycosis in a neonate presenting as necrotizing enterocolitis (NEC). Eur J Paediatr Surg 2008;18(2):119-20.

10. Nichol PF, Corliss RF, Rajpal S, et al. Perforation of the appendix from intestinal mucormycosis in a neonate. J Paediatr Surg 2004;39(7):1133-5.

11. Christenson JC, Shalit I, Welch DF, et al. Synergistic action of amphotericin B and rifampicin against rhizopus species. Antimicrob Agents Chemother 1987;31(11):1775-8.

12. Ozturk MA, Akin MA, Deniz $K$, et al. Neonatal gastrointestinal mucormycosis in an asphyxiated premature newborn. Turk J Paediatr 2011;53(6):705-8. 